\title{
Compact-sized excimer laser with two-side preionization
}

\author{
S.M. Baschenko, L.S. Marchenko \\ Institute of Physics, NAS of Ukraine, 46, prospect Nauky, 03028 Kyiv, Ukraine \\ E-mail: baschenk@iop.kiev.ua
}

\begin{abstract}
Compact-sized monopulse excimer laser with the foot-print equal to only $250 \times 80 \mathrm{~mm}$, total height $250 \mathrm{~mm}$ and beam axis height $50 \mathrm{~mm}$ was designed. Output energy at the wavelength $308 \mathrm{~nm}$ ( $\mathrm{XeCl}^{*}$ mixture) is $5 \mathrm{~mJ}$, maximal repetition rate $-1-2$ pulses per second, beam divergence -8 milliradians. The small foot-print, small axis height of beam in combination with low weight of laser $(5 \mathrm{~kg})$ allow this laser to be used in various optical schemes, where traditional, typical bulky ecximer lasers are unapplicable. Moreover, small dimensions, low weight in combination with low energy consumption (a few watts) allow them to be used in clear "field" conditions. Beside abovementioned characteristics, the designed laser differs from the traditional ones by two-side preionization that has a "spark" character near the "hot" massive electrode and the so-called "capacitive" or "barrier" one under the ground grid. This two-side preionization provides both igniting and sustaining of wider discharge thus allowing to reach beam with higher symmetry - its cross-section is $15 \times 20 \mathrm{~mm}^{2}$.
\end{abstract}

Keywords: excimer laser, preionization, dimension, weight.

Manuscript received 17.09.10; accepted for publication 02.12.10; published online 30.12.10.

\section{Introduction}

Excimer lasers possess yet unquestionable and mutchless advantage: highest output peak power in DUV-UV region of spectrum. But concomitant circumstance is low laser beam quality: high divergence (milliradians) of radiation and its wide spectrum (tens of $\mathrm{cm}^{-1}$ ) [1]. Besides, the very low impedance of excimer active medium results in contracting discharge and, therefore, the beam cross-section narrows and stretches [2]. In addition, the latter leads to a difference in beam divergences for X-Y axes and, as a consequence, to both difficulties and low efficiency in practical implementation of beam-matter interaction under focusing. To overcome this disadvantage, excimer laser producers were forced to design the so-called beam "homogenizer" that is quite complex and not so cheap.

Besides, one characteristic feature more of an excimer laser is its bulkness: large dimensions and high weight. Even the so-called (by the producer itself) "mini" excimer laser has dimensions $470 \times 250 \times 250 \mathrm{~mm}$ and weight up to $38 \mathrm{~kg}$ (EX5 by Gam Laser Inc.) or $300 \times 260 \times 210 \mathrm{~mm}$ and weight $36 \mathrm{~kg}$ (PSX-100 by MPB Inc.) [3]. It is evident that there exist many fields of investigations where these bulky devices cannot be implemented, although in some of these fields even a few laser pulses may be enough to obtain result due to high sensitivity of modern photodetectors.

\section{Construction and results}

A common external look of the designed laser (without any case) in front of traditional, typical one, its construction and electrical scheme are shown in Photo 1, Figs 1 and 2, accordingly. The dielectric tube $(50 \mathrm{~mm}$ outer diameter and $240 \mathrm{~mm}$ length) is the base of its construction. All the electrodes - main and subsidiary are placed inside the tube. A high voltage pulse generator (the so-called "invertor") is placed closely around the tube to minimize discharge loop inductance. Optical elements - both adjustable aluminium mirror and silica output window - are mounted on flanges at the tube ends. As Fig. 1 shows, preionization occures simultaneously from both sides. First (traditional) preionization - "sparky" near the "hot" massive electrode - is fulfilled by mean of additional spark electrodes placed at 3-mm distance from the "hot" electrode at both its sides and 15-mm distance from each other and in the chess-board order. The length of main electrode is $190 \mathrm{~mm}$, length of discharge $-170 \mathrm{~mm}$. Second preionization - the so-called "capacitive" or "barrier" from under "ground" grid electrode - is 
fulfilled by mean of additional metal plate electrode enclosed in several layers of mailar film. The length and width of plate electrode are $170 \mathrm{~mm}$ and $15 \mathrm{~mm}$, accordingly. The distance between grid and plate electrodes was adjusted experimentally and found to be optimal and equal to $2.5 \ldots 3 \mathrm{~mm}$. Principles of a pulse generator, both types of preionization action have been widely considered in literature [e.g., 2].

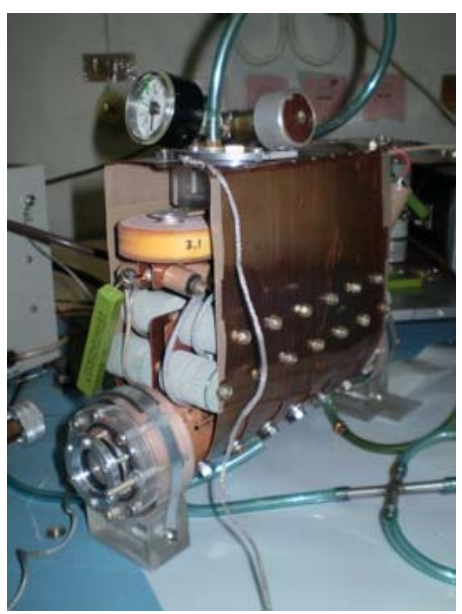

Photo 1. External look of the designed laser.

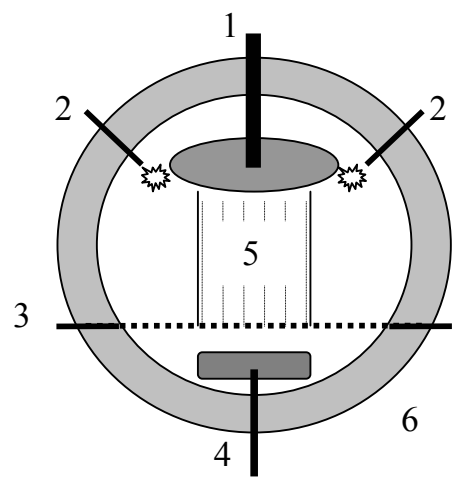

Fig. 1. Construction of the designed laser: 1 - "hot" massive electrode, 2 - subsidiary "spark" preionization electrodes, 3 "ground" grid electrode, 4 - subsidiary metal plate isolated electrode of "barrier" preionization, 5 - volume electrical discharge $15 \times 20 \times 170 \mathrm{~mm}^{3}, 6$ - dielectrical tube.

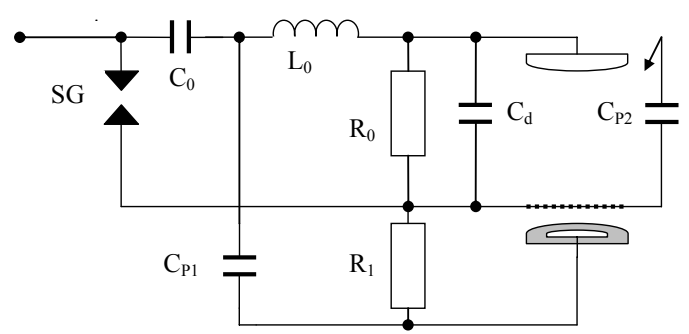

Fig. 2. Electrical scheme of the designed laser: SG - "spark" gap, triggering voltage is tuned by pressure in the gap and distance between electrodes; $C_{0}-$ main storage capacitor $10 \mathrm{nF} ; L_{0}$ - discharge inductance $20 \mathrm{nH} ; R_{0}$ - charging resistor $100 \mathrm{k} ; C_{d}-$ main discharge capacitor $5 \mathrm{nF} ; C_{p 2}-$ capacitor of "spark" preionization $2 \mathrm{nF} ; C_{p 1}-$ capacitor of "barrier" preionization $0.5 \mathrm{nF} ; R_{1}-$ resistor of "barrier" preionization $100 \mathrm{k}$.

The charging high voltage was up to $20 \ldots 23 \mathrm{kV}$, the role of a commutator was played by a passive sparky gap. The discharging voltage was adjusted previously by pressure in the gap and remained changeless under operation. For $\mathrm{He}: \mathrm{Xe}: \mathrm{CCl}_{4}$ gas mixture $\left(\mathrm{XeCl}^{*}, 308 \mathrm{~nm}\right)$ at the pressure $1 \mathrm{~atm}$, the output energy was equal to $5 \mathrm{~mJ}$. The laser beam cross-section was $15 \times 20 \mathrm{~mm}^{2}$, the beam divergence measured using a long-focus planeconvex lens along $\mathrm{X}, \mathrm{Y}$-directions was $6 \times 8$ milliradians. The maximal repetition rate reached up to $1 . .2$ pulses per second.

It ought to be mentioned that main efforts of authors was directed to laser size minimization, and electrical scheme is not optimal by its efficiency. Optimization of the electrical curcuit and gas mixture will be our next step in designing this laser.

\section{References}

1. A.V. Eletskiy // Uspekhi Fizicheskikh Nauk, 2(2), p. 279-314 (1978), in Russian.

2. V.Yu. Baranov, V.M. Borisov, Yu.Yu. Stepanov, Electro-discharge Excimer Lasers. Energoatomizdat, Moscow, 1988 (in Russian).

3. Laser Focus World, Optoelectronics Industry Sourcebook, 2004 Buyers Guide, PennWell Corp., pp. 11, 257. 